\title{
Relación Universidad - Comunidad: expresión de responsabilidad social de sus protagonistas
}

\section{University - Community relationship: Expression of Social Responsibility of its Protagonists}

\author{
Noraida Garbizo Flores* \\ Centro Universitario Municipal "Hermanos Saíz Montes de Oca", Pinar del Río, Cuba \\ ORCID: https://orcid.org/0000-0003-3109-468X \\ Mayra Ordaz Hernández \\ Universidad "Hermanos Saíz Montes de Oca", Pinar del Río, Cuba \\ ORCID: https://orcid.org/0000-0003-4729-2197

\section{Alba Marina Lezcano Gil} \\ Centro Universitario Municipal "Hermanos Saíz Montes de Oca", Pinar del Río. Cuba \\ ORCID: https://orcid.org/0000-0001-8574-8859
}

Recibido 22-11-19 Revisado 15-01-20 Aprobado 12-03-20 En línea 08-05-20

*Correspondencia

Email: norita@upr.edu.cu

\section{Citar como:}

Garbizo, N., Ordaz, M., \& Lezcano, A. (2020). Relación Universidad - Comunidad: expresión de responsabilidad social de sus protagonistas. Propósitos y Representaciones, 8(2), e412. doi: http://dx.doi.org/10.20511/pyr2020.v8n2.412 


\section{Resumen}

La visión de las universidades en el ámbito de la responsabilidad social, la ha convertido en una institución de referencia en la comunidad, propiciadora de relaciones, alianzas y diálogos de saberes, desdibujados de las nociones tradicionales que acentúan el rol exclusivo de formación hacia el interior de los predios universitarios. Desde esta perspectiva de análisis, se trata de asumir el vínculo universidad - comunidad como soporte de la formación integral del estudiante y eje articulador de sus procesos sustantivos. Ante el reconocimiento de esta trascendente relación, el artículo se orienta a realizar una revisión conceptual y análisis crítico del nexo universidad comunidad, expresión de responsabilidad social de sus protagonistas, con el empleo del método histórico - lógico y el auxilio del análisis documental. Los hallazgos apuntan a resaltar el vínculo universidad -comunidad como escenarios por excelencia en la formación integral de profesionales, ciudadanos y personas comprometidas con el desarrollo sostenible del entorno comunitario.

Palabras clave: Responsabilidad; Comunidad; Relación escuela comunidad; Necesidad social, Participación comunitaria.

\section{Summary}

The vision of universities in the field of social responsibility has made it a reference institution in the community, promoting relationships, alliances and dialogues of knowledge, blurred of traditional notions that accentuate the exclusive role of inward training of the university grounds. From this analysis perspective, it is about assuming the university-community link as a support for the integral formation of the student and articulating axis of their substantive processes. Given the recognition of this transcendent relationship, the article aims to carry out a conceptual review and critical analysis of the university-community nexus, expression of social responsibility of its protagonists, with the use of the historical-logical method and the help of documentary analysis. The findings aim to highlight the university-community link as scenarios par excellence in the comprehensive training of professionals, citizens and people committed to the sustainable development of the community environment.

Keywords: Responsibility; Community; School Community Relationship; Social Need: Community Participation.

\section{Introducción}

La Agenda 2030 para el Desarrollo Sostenible, aprobada en septiembre de 2015 por la Asamblea General de las Naciones, en su objetivo 4 declara: la necesidad asegurar que todos los alumnos adquieran conocimientos teóricos y prácticos necesarios para promover el desarrollo sostenible, mediante la educación para el desarrollo sostenible y los estilos de vida sostenibles, los derechos humanos, la igualdad de género y la promoción de una cultura de paz y de no violencia (p.27).

Ante el reconocimiento de esta necesidad, emerge la relación universidad comunidad como factor clave en la formación de profesionales que tributen al desarrollo sostenible, desde "el saber y saber hacer" de ciudadanos comprometidos y conocedores de su realidad social. Se trata pues de la concepción de un proceso de formación que se retroalimente de los saberes comunitarios y patrimoniales del entorno local, propiciando a su vez la participación social de la comunidad en su auto desarrollo con criterio de sostenibilidad, en alianza estratégica con la universidad.

En el devenir por el que se transita, se puede aseverar que: "los tiempos actuales requieren de una institución universitaria comprometida con la vida comunitaria, con las necesidades de En correspondencia con esta idea, incursionar en el estudio de la relación universidad - comunidad, deviene en elemento nuclear para concebir acciones de formación, investigación y extensión 
universitaria que partan de las problemáticas sociales, culturales, económicas y ambientales identificadas en el entorno comunitario, e integren cada vez más la universidad a la solución y/o mitigación de sus problemas.

Por consiguiente, este análisis nos adentra en el camino de educar en la comunidad y con la comunidad de manera socialmente responsable. Ante este panorama las preguntas obligadas son:

- ¿Constituye el vínculo universidad- comunidad un recurso potente para aprovechar la diversidad presente en las aulas universitarias y sacar partido de ella?

- ¿Las alianzas estratégicas entre la universidad y la comunidad son realmente efectivas en la formación profesional?

- ¿Cómo potenciar la participación de los estudiantes en proyectos comunitarios con la participación social efectiva de sus pobladores?

Cada una de estas interrogantes puede tener variadas respuestas y suscitar una multiplicidad de estudios con variadas aristas por explorar. Por ello, se requiere una visión transformadora del proceso de formación profesional, sostenido en la relación universidad comunidad.

Desde esta perspectiva de análisis, se coincide con Castilla y Inciarte (2015), al declarar:

La necesidad de una interrelación de carácter dialógico en la cual los universos interactuantes no siempre requieren la modificación de su identidad propia; pero si se debe desarrollar la capacidad de aprender organizacionalmente para pasar del discurso del deber ser a la acción comprometida de sus prácticas pedagógicas, investigativas y de servicio (...). (p.14)

Es evidente, la necesidad de romper la inercia, crear un tejido de interrelaciones diversas entre la universidad y la comunidad en calidad de escenarios por excelencia del proceso de formación, de manera que esta relación se convierta en eje transversal de los procesos universitarios para intencionar el vínculo de saberes y potenciar la responsabilidad social universitaria en la medida que se promueve la responsabilidad social comunitaria.

Por tanto, con la intención de aportar caminos que permitan hacer cada vez más efectiva el vínculo universidad -comunidad, emerge la necesidad de realizar una revisión de las posiciones teóricas y metodológica asumidas por diversos autores que se han dedicado a evaluar estas categorías y clarificar el tejió de relaciones complejas que se establecen entre ellas, de manera que la responsabilidad social de la universidad se materialice en estrategias que favorezcan la sostenibilidad de las comunidades.

\section{La universidad escenario de responsabilidad social en la comunidad}

La universidad cambia, cambian los escenarios formativos, aumenta la posibilidad de incorporar profesionales de otras ramas a dichos procesos, realiza cambios en los roles y abre oportunidades de acceso en general" (Veliz, 2013). Es indudable que: "esto exige que el compromiso de Instituciones de Educación Superior sea asumido como compromiso de responsabilidad social, cimentado en comportamientos éticos que den respuesta efectiva a los problemas, con soluciones responsables y oportunas" (Castro, Marín \& Senior 2016, p.185).

Desde esta concepción, "la función social de la educación juega un rol fundamental, al permitir la preparación de los ciudadanos a través del proceso de socialización. Asegurar mediante la asimilación y objetivación del individuo, trasmitir la herencia cultural de generación en generación" (Martí, Montero \& Sánchez, 2018, p.266). 
En esta línea de análisis, Eito \& Gómez (2013), esbozan que: "pensar y trabajar comunitariamente es un motor ideológico de transformación social, de abordar los problemas socialmente, de reforzar la participación de los interesados en la toma de decisiones profesionales y de reforzar la democracia" (p. 5).Por su parte, Lezcano (2018), declara que la problemática de una comunidad necesita de la contribución de sus pobladores y del tratamiento de sus instituciones, enfatiza que se puede hacer mucho para mitigar los problemas, desde el cambio de actitudes y hábitos en las comunidades.

En este sentido, un reto que devela la responsabilidad social universitaria, está dado por la necesidad de propiciar un relación armónica entre la universidad y la comunidad, como elemento clave que favorece el proceso formativo, a partir del diseño de acciones que potencien la participación social efectiva de estudiantes y profesores en proyectos, estrategias y programas que contribuyan a la solución de los problemas comunitarios con la participación activa de la comunidad, desde la comunidad.

Por ello, se coincide con Alarcón (2016) al insistir que la universidad tiene que ser innovadora porque: "es la universidad que se reforma permanentemente, enriqueciendo su modelo de gestión, para cumplir mejor su función social mediante la sinergia de las actividades de formación, investigación y la extensión universitaria, vinculadas siempre con la sociedad" (p. 6).

Se privilegia en este anisáis, la capacidad de la universidad de ser creativa, de abrir sus predios a las mejores experiencias comunitarias, corroborando que, "la intervención de la universidad en la comunidad requiere de métodos y procedimientos que permitan un accionar desde y de la propia comunidad" (Gómez, 2015, p.13). De esta manera la interrelación de la universidad con la comunidad cobra importancia para satisfacer las necesidades formativas a que se aspira socialmente (Mendoza \& Enebral, 2018).

Para Gómez (2015), lograr la sostenibilidad de las transformaciones requeridas en la comunidad, requiere de la implementación del método educativo para el desarrollo comunitario, procedimiento que muestra la lógica de un proceso complejo que permite el cambio, donde los actores sociales protagonizan un rol de autodesarrollo. Por tanto, "en los momentos actuales es cuando con más fuerza las instituciones universitarias deben repensar su accionar en la interrelación con la comunidad, a partir del reconocimiento del otro, sobre la base de la formación de la conciencia crítica y desde la participación autogestionaria” (Fernández, 2019, p.10).

En este escenario, según Tonon (2012):

cabe la universidad, considerada como institución social de referencia y pertenencia, un rol protagónico en el desarrollo de relaciones con la comunidad de la cual es parte, con el objetivo de configurar una nueva expresión de lo público en términos de relación entre los sujetos y de espacio de construcción de ciudadanía. (p.512)

Por tanto, se precisa de: "una educación crítica y solidaria engloba a todos los actores de la institución escolar (...) que trasciende los muros de la escuela para vincularse participativamente con el barrio, la ciudad, el país y el mundo (Betto, 2015, p.7).

De tal manera, el compromiso social de la universidad se expresa en la interrelación con la comunidad, desde una participación autentica y efectiva de sus protagonistas, elemento clave que va generando una presencia efectiva de la universidad en el contexto comunitario, a partir de la creación de acciones de cooperación y proyectos conjuntos que revelan que la comunidad es un escenario por excelencia de la formación profesional, personal y social y fuente de las mejores experiencias socioculturales y ambientales. 
Todo lo anterior destaca el valor de la extensión universitaria como uno de los procesos más integradores de las universidades, que facilita la integración de todos sus procesos y fortalece la relación universidad-sociedad, al convertirse en una alternativa para la intervención en las comunidades. La extensión es la actividad (...), que también contribuye a la transformación de la sociedad mediante su propia participación en el desarrollo cultural (González \& González, 2014, p.11).

\section{La comunidad, escenario de responsabilidad social}

Irrumpir en el estudio de la relación universidad -comunidad, implica reconocer los aportes de ambas entidades a la formación profesional, personal y social de los estudiantes, apoyados en el paradigma de formación integrar. Vale destacar que son disimiles las experiencias que evidencian el valor de la comunidad como escenario de formación, así como las iniciativas universitarias en pos de resaltar el valor de las comunidades en la tarea de educar para la vida.

León, Tamayo y Aguilar (2019) conciben: "la comunidad como un fenómeno multidimensional donde intervienen elementos geográficos, sociológicos, naturales, territoriales, políticos, culturales y sociales que deben ser conocidos, respetados e integrados para hacer de la localidad un organismo social eficiente y efectivo en lo material y espiritual" (p.91).

Mendoza \& Enebral (2018) sostienen que: "la comunidad constituye un mecanismo intermedio entre la sociedad y el individuo. En ella se materializan todas las interacciones del sujeto, ya que se concreta la relación sociedad-individuo" (p.4). Para Lezcano (2015), la comunidad no es estática, está sujeta a cambios como toda institución social.

En los estudios relativos a la comunidad como entidad social, se destacan los planteamientos de Pineda, González \& Pineda (2018), al destacar la comunidad como:

Grupo de seres humanos que tienen ciertos elementos en común: el idioma, las tradiciones, valores, tareas, ubicación geográfica (un barrio, por ejemplo), roles, estatus social y hasta necesidades crea una identidad, mediante la diferenciación de otros grupos o comunidades que es compartida y elaborada entre sus integrantes. (p.156)

Mucho pudiera abundarse en torno al término comunidad, concepto que ha venido reconfigurándose en el transcurso del desarrollo histórico social, apreciándose definiciones variadas en las que se puede advertir, la concepción de espacio presente, la existencia de un asentamiento poblacional, su carácter objetivo, subjetivo y psicológico y sus aspectos físicos, sociopolíticos económicos, psicopedagógicos y culturales. En esta línea de análisis y con énfasis en la "comunidad del término comunidad", Sánchez (2005), destaca los términos: localidad compartida, interacción social y relaciones y lazos comunes.

De igual manera, el valor educativo de la comunidad ha constituido tema de análisis por numerosos investigadores del tema. En este sentido, Gonzales (2003) señala:

La educación en la comunidad debe centrar la atención principal en el hombre, en sus dimensiones personales, singularidad, originalidad, autonomía, libertad de opción y trascendencia; enfatiza en el desarrollo y rescate de la cultura, los valores y compromisos e identidad con la comunidad, con sus éxitos y sus dificultades, en una actitud responsable, aptos para enfrentar los nuevos retos, como sujetos activos para el cambio (p.30).

Mendoza \& Enebral (2018), al evaluar el proceso de socialización en el ámbito comunitario, sostienen que: "es a nivel de comunidad donde cada individuo recibe de manera 
singular y simultánea las influencias sociales como inmediatas. Y es ahí donde el hombre actúa de manera individual o colectiva, reflejando los aspectos sociales más generales” (p.4).

En estos análisis no se puede obviar lo planteado por Báxter (2007), al señalar que: "en la comunidad se desarrolla un proceso educativo mucho más espontáneo, con características asistémicas no formales, donde se pone énfasis en tradiciones y costumbres, formas de vida y normas de conducta (p.104)

De ahí que lo educativo-comunitario penetra en la complejidad del tejido social, presupone un estudio en lo individual y lo social comunitario; el primero revela las características de los sujetos portadores de la acción educativa, la interactividad que genera un proceso de enseñanza-aprendizaje, mediado por sus propias necesidades y acciones que dinamizan el cambio en la comunidad (Gómez, 2015, p.10). En tal sentido, se coincide con Villamañan (2016) al destacar que los vínculos comunitarios son aquellos concebidos como simetría o equidad social.

Las consideraciones anteriores, apuntan hacia el valor educativo de la comunidad, centrado en la valía de su gente, sus tradiciones, relaciones y aportes a la cultura y la consolidación de una historia que sirve de legado y continuidad para las nuevas generaciones. En la comunidad se aprende participando, asumiendo retos, involucrando a las instituciones que en ella se encuentran, lo que facilita el proceso de autogestión comunitaria.

En este contexto se coincide con Fernández (2019) al expresar que:

Se pretende fortalecer la identidad en los que se involucran en este proceso, que otros actores sociales comunitarios participen en el proceso educativo, que se trascienda lo legislado, al modificar las interpretaciones y atribuciones que se hacen en relación con la participación desde una práctica consciente, para lograr el fin que se persigue (p.30).

$\mathrm{Al}$ asumir estas consideraciones, derivadas de la sistematización teórica de los estudios consultados, se hace necesario repensar el peso ordenador que tienen el contexto comunitario en la formación profesional y ciudadana del estudiante universitario. Por tanto, se revela la significación de la responsabilidad social comunitaria, entendida como participación y compromiso de los miembros de la comunidad, las organizaciones, entidades e instituciones en la definición e implementación de acciones que tributen al crecimiento personal de sus pobladores y el desarrollo sostenible de la comunidad.

De este modo, la responsabilidad social de la comunidad en el ámbito universitario se expresa en la proyección de acciones desde la comunidad en apoyo a los procesos sustantivos que la universidad desarrolla. La comunidad, escenario educativo por excelencia puede y debe contribuir con la institución universitaria, a partir de la colaboración conjunta en proyectos, estrategias y planes de acciones que surjan de la comunidad e involucren a la comunidad universitaria.

Para alcanzar tales propósitos, fortalecer la relación universidad - comunidad, desde el protagonismo responsable de ambas comunidades, se erige como factor clave para potenciar transformaciones en estos contextos.

\section{La relación universidad - comunidad: retos y oportunidades}

El abordaje de la relación universidad - comunidad, ha sido ampliamente abordada por diversas disciplinas científicas entre las que puede citarse, la Pedagogía, la Sociología y la Psicología Educativa, en correspondencia con el objeto de estudio, resultando común entre ellas, el valor de ambas entidades para el proceso de formación profesional, la socialización y la educación no 
formal e informal que se sucede en este contexto y tienen a su base las históricas relaciones escuela-comunidad.

En las prácticas académicas, sobre el flujo interactivo universidad - comunidad se señalan múltiples categorías o nociones conceptuales y procedimentales asociadas: Integración, asociación, complementariedad, impacto, conexión, cooperación, involucramiento, unión, acompañamiento, mediación, articulación, elaboración de convenios, redes y alianzas; entre otros (Castilla \& Inciarte, 2015, p.4).

Estas categorías refieren las acciones que se implementan entre ambas entidades y favorecen su autodesarrollo. Según Socarras (2005), "el campo comunitario, en lo particular, ha constituido escenario común de multiplicidad de experiencias donde lo participativo constituye reclamo, y hasta razón de ser y, al mismo tiempo, de por sí, es el campo más estrechamente vinculado a lo cotidiano" (p.78). Es una realidad, apunta Murga (2017), "que la transición hacia la sostenibilidad está sin duda en marcha. Y la Universidad no es ajena a este gran fenómeno de transformación integral y global que involucra a todo tipo de actores" (p.63).

En relación con lo planteado por los autores citados, la comunidad se privilegia con la implementación de buenas prácticas que atienden de manera particular la problemática ambiental, económica y sociocultural y favorecen la implementación de programas, que resaltan sus valores, tradiciones y potencialidades para asumir transformaciones y potenciar el desarrollo comunitario. Por ello, "se impone el conocimiento de los componentes fundamentales de lo comunitario: la colaboración, la participación social y el proyecto colectivo que se gesta socialmente (...) “(Villamañan, 2016, p.498).

De este modo, la relación universidad - comunidad, es inoperante sin el conocimiento de la cultura organizativa de una comunidad. Según Dávalos (2005), es un requisito importante para promover una participación efectiva y hacer de ella un ejercicio democrático, a través del cual se eduque a distintos sectores de una comunidad en una sociedad que aspire a establecer una gestión eficiente de los recursos en el marco de una adecuada autonomía para su desarrollo.

Al respecto, resultan muy sugerentes las consideraciones de Tréllez (2015), al subrayar que el tema buenas prácticas tiene que ver la inclusión de: participación, intersectorialidad, interculturalidad, nexos entre los aspectos naturales y sociales, la articulación escuela-comunidad, el arte, la investigación, la innovación y otros que resultan de gran interés para generar actitudes transformadoras en la comunidad, como factor de éxito.

En correspondencia con estas ideas y ante la preocupación creciente que ha tenido la necesidad de lograr un nexo bidireccional entre universidad -comunidad, Valdés y Villegas (2017), destacan las potencialidades de la estrategia sociocultural, como herramienta de trabajo, al subrayar que:

Una estrategia de gestión sociocultural quedaría limitada solamente a la acción si no se involucrara a los actores sociales que participan en ella, como facilitadores, para que intervengan protagónicamente en esos procesos que son también los que construyen el patrimonio como una de las principales expresiones de identidad del capital social que lo genera. (p.53)

Ante el reconocimiento del valor metodológico de la aludida relación y la necesidad de lograr la sostenibilidad de las iniciativas de desarrollo local en las comunidades, es preciso considerar que "las acciones de motivación, sensibilización, información y formación, son herramientas muy útiles (...), de ahí que las estrategias de desarrollo local hayan ido asumiendo 
una perspectiva generacional, concretada en políticas y programas que atienden esa dimensión" (Iglesias \& Jiménez, 2017, p.64).

Por lo antes descrito, es necesario visibilizar la participación como elemento nuclear en la concepción de los procesos comunitarios, sin crear la falsa ilusión de considerar la presencia física en las actividades que se desarrollan como indicador que evidencia que las comunidades están preparadas para participar de manera efectiva en su autodesarrollo. Se necesita clarificar el término participación para poder lograr su verdadera expresión en los procesos comunitarios.

\section{Participación social. Factor de éxito.}

Por las riquezas de las interacciones que tienen lugar en su interior y por la diversidad de actores sociales que alberga, la universidad y la comunidad, devienen como espacio de transformación y cambio social por excelencia (Garriga, 2010).

Se reconoce, que la universidad para implementar transformaciones en la comunidad, es fundamental partir de ella misma, sus necesidades, intereses, motivaciones, entro otros indicadores, lo cual asegura la sostenibilidad de los cambios. Por tanto, la relevancia de la participación en los procesos de transformación comuntaria, se erige como tema esencial en el debate contemporáneo, lo cual requiere prestar especial atención a las necesidades, intereses, recursos actuales y potenciales de la comunidad.

Según D’Angelo (2010):

(...)cuando hablamos de participación, estamos hablando de algo más que estar presente, más que movilizarse, más que intercambiar criterios, más que opinar, significa sensibilizarse, tomar parte, implicarse, decidir y actuar comprometidamente, contribuyendo así a la implementación de estrategias que resulten legitimas y sostenibles en la solución de problemas comunes. (p. 54)

Para Pineda, González \& Batista (2019):

La participación que tienen los actores sociales (...) tendrá sentido si en cada barrio, en cada cantón, en cada comuna, los ciudadanos y por ende sus organizaciones se proponen como meta asumir una aptitud autogestionaria conjugando el capital económico con el capital social y humano. (p.161)

En relación con el estado del arte de la participación como elemento secular de los procesos de transformación social, se precisa repensar las formas de participación predominantes, la implicación de los sujetos en la identificación de los problemas, la propuesta de soluciones, así como el diseño de las acciones que se proponen en este contexto.

Está claro que en la medida que se estimule la participación social, sostenida en la responsabilidad de sus protagonistas se potenciará el desarrollo humano. Esto significa que el desarrollo humano se logra a través de la participación consciente del sujeto en la transformación de la sociedad (Martínez, Marrero \& Núñez, 2016).

Desde el prisma de la responsabilidad social, es evidente que la participación social se muestra como un proceso transformador, sistémico y articulado con el trabajo comunitario, precisamente: "su eficacia está precisamente en la unión de la comunidad, en su organización y en el papel que desempeñan los líderes del grupo" (Vázquez, Rojas \& Sánchez, 2018, p.184).

En consonancia con lo planteado, resultan muy sugerentes los planteamientos de Galeana y Sáinz (2006), quienes destacan como elementos esenciales para potenciar la participación: acción colectiva, permitir a los individuos convertirse en protagonistas de las transformaciones y la necesidad de construir una dimensión básica del desarrollo local. 
Desde esta concepción, abordar la participación para la comunidad implica "el reconocimiento de poder establecer una participación real que implica: ser constructiva, popular y emancipadora; una participación que pueda convertirse en un mecanismo efectivo de socialización" (Dávalos, 2005, p.52).

Al respecto, Iglesias \& Giménez (2017) refieren que: "la participación social efectiva de la comunidad se traduce en el acceso y la presencia real de los individuos y los grupos en las instituciones y organizaciones y la posibilidad de intervenir en las decisiones" (p.38). En tal sentido, la participación determina el carácter activo del sujeto, al intervenir en las decisiones sociales (Altavaz, 2014).

Se privilegia en estos análisis las consideraciones de Obret, Céspedes y Hernández, (2015) al señalar que:

La participación social, aspecto muy vinculado con la autonomía y la responsabilidad, va a permitir la transmisión y el aprendizaje de unos valores que van a contribuir a la promoción de una ciudadanía que abogue por la construcción de comunidades democráticas más justas y equitativas. (p.8)

No cabe duda, que potenciar la participación social en la comunidad es un proceso complejo, que tiene un componente cultural que tranversaliza este proceso e incide en las diversas formas de participación comunitaria, incidiendo de manera particular, sus rasgos distintivos, historia, la forma en que se asumen las problemas que le atañen y la manera en que se involucran en su autogestión, razón que apunta a la necesidad de fomentar una cultura de participación.

En este sentido, se coincide con Rubio \& Vera (2012) al destacar que:

La cultura participativa indica una conducta socialmente activa frente al conflicto, demanda o problema, que afecte a las personas en su cotidianeidad; indica un nivel sostenido y coherente de acciones concretas. Es la capacidad o potencialidad endógena de los grupos para ejercer el control, tomar decisiones y emprender acciones de cambio, favorables al desarrollo social y cultural de la comunidad. (, p.9)

Desde estos referentes, potenciar una participación social efectiva en la universidad, favorece la formación de profesionales que descuellen por su autonomía y toma de decisiones en diversos contextos, de acuerdo al modelo de formación profesional. Por su parte, en la comunidad, la participación social efectiva se traduce en la presencia real de sus pobladores en la identificación y solución de los problemas de este contexto, al potenciar su autodesarrollo. En correlato a lo expresado, en la sistematización teórica de tema, se identifican una serie de elementos comunes que devienen en regularidades del proceso de participación, válidas para el contexto comunitario y universitario, entre las que se destacan:

- El carácter polémico y abarcador de la participación social.

- La participación como instrumento de socialización en la comunidad.

- Las diferentes formas de participación social, sustentadas en una dimensión sociocultural.

- La participación social efectiva, resultado de la presencia real de los individuos en los procesos que le son inherentes.

- El rol protagónico de la participación en la autoeducación en la educación formal y no formal 


\section{Reflexiones finales}

En la actualidad, el proceso de formación de profesionales trasciende de ser una formación centrada en las competencias y habilidad que se desarrollan hacia el interior de las universidades para operar con el currículo, a ser un proceso de perfil amplio, sustentado en el paradigma de formación integral, que se soporta en la relación universidad - comunidad como escenario de confluencia entre los saberes académicos y patrimoniales que favorecen la participación social efectiva en los procesos autogestionarios.

Una realidad es incuestionable, la universidad se transforma, se perfeccionan los procesos de formación y se transforman las comunidades con la asunción protagónica de responsabilidades en los procesos que conducen al desarrollo sostenible de manera global y comunitaria. De este modo, se considera esencial, revelar el significado de la responsabilidad social comunitaria, como elemento nuclear en la concepción de los procesos comunitarios y factor de éxito en el desarrollo de acciones coordinadas con las universidades.

En este panorama, es necesario visibilizar la importancia de la participación social como instrumento de transformación social, visto desde una dimensión integral, que privilegia lo sociocultural como punto de partida para identificar sentidos y formas de participación comunitaria. El reconocimiento del carácter complejo de este proceso se reconoce en el abordaje teórico y metodológico de este proceso, referente que develan la necesidad de profundizar en estos estudios, desde la perspectiva del desarrollo sociocultural de las comunidades y las transformaciones que tienen lugar hacia su interior.

\section{Referencias}

Alarcón, R. (2016). Universidad innovadora por un desarrollo humano sostenible: mirando al 2030. Transformación, 12(1), 1-8. Recuperado de: https://transformacion.reduc.edu.cu

Altavaz, A. (2014). La formación ciudadana y el desarrollo de la personalidad. VARONA, Revista Científico-Metodológica, 59(1), pp.23-28.

Báxter, E. (2007). Educar en valores. Tarea y reto de la sociedad. La Habana: Pueblo y Educación.

Betto, F. (2015). Educación crítica y protagonismo cooperativo. Conferencia dictada en el Congreso Internacional "Pedagogía 2015". La Habana, Cuba.

Castilla, C., \& Inciarte A. (2015). Currículo y Comunidad en la Educación Superior. Ponencia presentada en el Pos Doctorado en Ciencias Humanas. Universidad de Zulia Venezuela.

Dávalos, R. (2005). La participación en la comunidad. La Habana: Editorial Caminos.

D’Angelo, O. (2010). Marco teórico metodológico aplicado: Desarrollo de subjetividades y espacio de participación comunitaria para la transformación social. La Habana: CIPS.

Eito, A., \& Gómez, J. (2013). El concepto de comunidad y el Trabajo Social. Revista Espacios Transnacionales, $\quad 1, \quad 132 \quad-\quad 145 . \quad$ Recuperado de http://www.espaciostransnacionales.org/conceptos/conceptotrabajosocial

Fernández, A. (2019). La interrelación instituciones universitarias - comunidad, un modelo participativo que lo promueve. Transformación, 15(1), 27-38. Recuperado de http://scielo.sld.cu/pdf/trf/v15n1/2077-2955-trf-15-01-27.pdf

Galeana, O.S., \& Sáinz Villanueva, J.L. (2006). La participación social: un horizonte posible para el desarrollo local. Revista Trabajo Social, 2(4), 113-123.

Garriga, T. (2010). La autonomía integradora. Una alternativa teórico metodológica para el abordaje de los procesos comunitarios. La Habana: Acuario.

Gómez, A.R. (2015). La universidad y su rol participativo a través del método educativo de intervención social. Revista Congreso Universidad, 4(1), 1-15. Recuperado de: http://www.congresouniversidad.cu/revista/index.php/congresouniversidad/index. 
González, M. (2003). Desarrollo comunitario sustentable. Propuesta de una concepción metodológica en Cuba desde la Educación Popular. (Tesis de doctorado en Ciencias de La Educación). La Habana, FLACSO.

González, G.R., \& González, M. (2014). Extensión universitaria. El arte de promover cultura. Curso impartido en el 9no Congreso Internacional de Educación Superior "Universidad 2014", Palacio de las Convecciones de la Habana, Cuba.

Iglesias, M., \& Jiménez, R. (2017). Desarrollo local y participación social. ¿De qué estamos hablando? Revista Estudios del Desarrollo Social: Cuba y América Latina, 5(1), 60-73. Recuperado de http://scielo.sld.cu/pdf/reds/v5n1/reds05117.pdf.

León, M. A., Tamayo Valdés, M. C., \& Aguiar, A. I. (2019). El trabajo con el texto en la comunidad en la ciudad de Cienfuegos. Universidad y Sociedad, 11(3), 88-95. Recuperado de http://rus.ucf.edu.cu/index.php/rus

Lezcano, A. (2015). Estrategia de educación ambiental en la comunidad Raúl Maqueira del municipio Consolación del Sur en la provincia Pinar del Río. (Tesis presentada en opción al Título Académico de Máster en Gestión Ambiental). Universidad de Pinar del Río Hermanos Saíz Montes de Oca, Cuba.

Lezcano, A.M., Suero, L., \& Garbizo, N. (2018). Proceso de gestión de la educación ambiental comunitaria. Apuntes para un debate. Revista Atlante. Cuadernos de Educación y

Desarrollo, 41-15. Recuperado de https://www.eumed.net/rev/atlante/2018/10/educacionambiental-comunitaria.

Martínez, Y., Marrero, R. \& Núñez, A. (2016). La participación y el protagonismo en el contexto escolar. Ponencia presentada en el Congreso Internacional de Investigadores sobre Juventud. Palacio de Convenciones de La Habana, Cuba.

Martí Chávez, Y., Montero Padrón, B., \& Sánchez, K. (2018). La función social de la educación: referentes teóricos actuales. Revista Conrado, 14(63), 259-267. Recuperado de http://conrado.ucf.edu.cu/index.php/conrado.

Mendoza, M.M., \& Ebebral, R. (2018). El perfeccionamiento de la interacción escuela comunidad, desde las actividades pedagógicas. Atlante. Cuadernos de Educación y

Desarrollo, 1, 1-15. Recuperado de https://www.eumed.net/rev/atlante/2018/02/interaccionescuela-comunidad.html

Murga, M. (2017). Universidades en transición. Hacia una transformación institucional orientada al logro de la sostenibilidad. Revista Iberoamericana de Educación, 73, 61-84. Naciones Unidas (2018), La Agenda 2030 y los Objetivos de Desarrollo Sostenible: una oportunidad para América Latina y el Caribe (LC/G.2681-P/Rev.3), Santiago.

Obret, R., Céspedes, E., \& Hernández, R. (2015). La Participación Social Universitaria en la Integralidad de los jóvenes Cubanos. Ponencia presentada en el Congreso Internacional de Investigadores sobre Juventud. Palacio de Convenciones de La Habana, Cuba.

Pineda, M.R, González, Y. \& Batista, R. (2019). La gestión sociocultural como herramienta del desarrollo local. REDEL. Revista Granmense de Desarrollo Local, 3(1), 154- 163.

Rubio, D., \& Vera, V. Comunidad y participación social. Un debate teórico desde la cultura. Contribuciones a las Ciencias Sociales, 1, 1-15. Recuperado de http://www.eumed.net/rev/cccss/20/rmvv.html

Socarras, E. (2005). Participación, cultura y comunidad. En: N, Hernández (Ed.), Trabajo comunitario. Selección de lecturas (pp. 37-49). La Habana: Editorial Caminos.

Tréllez, E. (2015). Tréllez Solís Eloísa, (2015). Educación ambiental comunitaria en América Latina Lima, Perú (Contrato con PNUMA/ROLAC 002 Red de Formación Ambiental para América Latina y el Caribe.

Tonon, G. (2012). Las relaciones universidad-comunidad: un espacio de reconfiguración de lo público. Polis, Revista de la Universidad Bolivariana, (11)32, 511- 520.Recuperado de https://scielo.conicyt.cl/pdf/polis/v11n32/art24.pdf

Valdés, M.G \& Villegas, T. (2017), Responsabilidad social universitaria: fundamento para la Gestión Sociocultural, Revista Cubana de Educación Superior, 3, 55-62. Recuperado de http://www.rces.uh.cu/index.php/RCES/article/viewFile/186/229 
Vázquez, M., Rojas, B. \& Sánchez, A.G. (2018). Participación comunitaria de los jóvenes en Cuba para el desarrollo social. Estudios del Desarrollo Social: Cuba y América Latina, 10(2), 178, 204.

Véliz, J.A. (2013). Modelo de Gestión de la Extensión Universitaria para la Universidad de Ciencias Médicas de Pinar del Río: Estrategia para su implementación" (Tesis de Doctorado). Universidad de Pinar del Río, Cuba.

Villamañan Alba, M. (2016). Lo comunitario en las representaciones sociales de la violencia. Psicología \& Sociedad, 28(3), 494-504. doi: http://dx.doi.org/10.1590/180703102016v28n3p494 